\title{
Hacia un análisis de Sobre la interpretación 9: La crítica de Aristóteles a las posturas deterministas a la luz del análisis de los "futuros contingentes"
}

\author{
DIEGO TABAKIAN \\ Universidad de Buenos Aires \\ Universidad del Salvador
}

Resumen: Desde la Antigüedad, el capítulo 9 de Sobre la interpretación de Aristóteles ha sido objeto de múltiples discusiones interpretativas: desde los principios lógicos que allí están en juego hasta la concepción aristotélica del tiempo y la necesidad. En el presente trabajo, nos proponemos dilucidar los compromisos fisico-ontológicos de la teoría aristotélica de la verdad a partir del análisis del problema del valor de verdad de los enunciados sobre futuros contingentes. Nuestro abordaje de Sobre la interpretación 9 partirá de los conceptos de "causalidad", "necesidad" y "azar", tal como son conceptualizados en Física II. 1, 8, 9 y Metafísica V. 5 yVI.3. En efecto, entendemos que la dificultosa reconstrucción de los argumentos allí formulados se debe a que Aristóteles no es explícito acerca de la concepción determinista contra la que discute. Debemos remitirnos 
a dichos tratados para dilucidar la argumentación determinista y la crítica aristotélica a esta posición.

Palabras clave: determinismo, fatalismo, necesidad, verdad.

\title{
Towards an Analysis of De Interpretatione 9: Aristotle's Critique of the Determinist Theories in Light of the Analysis of Future Contingents
}

\begin{abstract}
Since Antiquity, Aristotle's De Interpretatione chapter 9 has been object of multiple interpretative discussions: from the logical principles involved in the argumentation to the Aristotelian conception of time and necessity. In this presentation, we analyze the problem of the truth-value of propositions about future contingent events in order to elucidate the physical and ontological presuppositions underlying Aristotle's conception of truth. Our interpretative strategy consist in analyze certain concepts of De Interpretatione 9 (such as "causality", "necessity", "chance", etc.) in correspondence with their explicit conceptualizations in Physics II. 1, 8, 9 and Metaphysics V. 5 y VI. 3. Since Aristotle criticizes his opponent without rendering explicit his deterministic conception, the arguments of De Interpretatione 9 are very difficult to reconstruct. We appeal to these treatises to elucidate the determinist argumentation and the Aristotelian critique.
\end{abstract}

Key-words: determinism, fatalism, necessity, truth.

\section{Introducción}

— n Sobre el alma el Estagirita afirma que las composiciones que efectúa el intelecto entre sujeto y predicado en el juicio se realizan mediante la cópula "ser" en tiempo pasado, presente y futuro (430b4-5), lo que sugiere que los enunciados declarativos son evaluables veritativamente con independencia de su formulación temporal, es decir, sin importar si su objeto ocurre en un tiempo pretérito, actual o futuro. Aristóteles aborda indirectamente esta cuestión en Sobre la interpretación 9 cuando expone el espinoso problema del valor de verdad de los enunciados sobre futuros contingentes y se pregunta si la verdad de las predicciones implica la ocurrencia necesaria de su objeto.

El debate hermenéutico acerca del problema de los "futuros contingentes" en Sobre la interpretación 9 es sumamente vasto: a partir de la Anti- 
güedad ${ }^{1}$ la discusión ha girado en torno a los principios lógicos ${ }^{2}$ involucrados en la argumentación dialéctica y a la concepción aristotélica del tiempo y la necesidad. ${ }^{3}$ Entre los especialistas no hay acuerdo acerca de cuál es la problemática abordada en Sobre la interpretación 9 ni de cuál es la posición que Aristóteles discute en un contexto eminentemente dialéctico. Las interpretaciones en pugna identifican esta posición o bien con el fatalismo o bien con el determinismo causal. A grandes rasgos, la primera problemática refiere a la compatibilidad entre el presupuesto de que todos los enunciados son o bien verdaderos o bien falsos con la creencia en la libertad de nuestros actos. En este sentido, se ha argumentado que el fatalismo no tiene que ver con la causalidad, sino con los valores de verdad de los enunciados: si una predicción es verdadera, ello implica que el evento predicho es necesario (en el sentido de inevitable). Por otro lado, la segunda problemática consiste en el intento de reconciliar la creencia generalizada de que todos los eventos se encuentran causalmente determinados con la opinión de que podemos actuar libremente, es decir, que podríamos haber evitado actuar como efectivamente lo hicimos (White 1981: 231). En otras palabras, el fatalismo se distingue del determinismo causal en que afirma únicamente que la verdad (de un enun-

${ }^{1}$ De acuerdo con el testimonio de Simplicio (CIAG 407: 6-13), los peripatéticos sostuvieron la interpretación tradicional y también, de acuerdo con Boecio (CIADI 1880: 208), los estoicos. Algunos filósofos y comentadores de Aristóteles (como Epicuro, Nicóstrato y Alejandro de Afrodisia) sostuvieron esta lectura porque, argüían, era el único modo de evitar el determinismo. Sobre estas posiciones, véase Cicerón, Sobre el destino IX 18, X 21, XVI 37-8, Sobre la naturaleza de los dioses I 25 70; Simplicio, Comentario a las Categorías de Aristóteles (406 13-16) y Alejandro de Afrodisia, Sobre el destino 10 (1777-1787), 16 (187 22), 17 (188 3) y 27 (197 12-17). Otros filósofos (como Carnéades, Crisipo de Solos y Cicerón) rechazaron esta posición. Véase Cicerón, Sobre el destino X 20-1, XII 27-8, XVI 38; Diógenes Laercio, VII 65 y Pseudo Plutarco Sobre el destino 574. Para un completo estado de la cuestión de estas posiciones, véase Sorabji 1980: 92-94.

2 Entre los antiguos que sostuvieron la interpretación tradicional, hubo un consenso generalizado de que el principio lógico en discusión en Sobrela interpretación 9 era el Principio de Bivalencia (PB): "todo enunciado es o bien verdadero o bien falso". A partir de J. Łukasiewicz 1970 y 1957 se incorporó al análisis textual y a la discusión interpretativa el Principio del Tercero Excluido (PTE). De acuerdo con la formulación tradicional, el PTE enuncia que cuando dos proposiciones se encuentran opuestas contradictoriamente no pueden ser ambas falsas. En la formulación moderna el principio del tercio excluso constituye la tautología p v $\neg$ p, es decir, "una disyunción compuesta de proposiciones contradictorias es necesariamente verdadera”. Finalmente, Whitaker (1996) destacó en la argumentación fatalista la función de la Regla de Pares Contradictorios (RPC): "En una disyunción compuesta de proposiciones contradictorias, una de ellas es necesariamente verdadera y la otra, necesariamente falsa".

${ }^{3}$ Sobre estas dos últimas cuestiones, véase especialmente Hintikka 1973, McKim 1972, Von Wright 1984, Sorabji 1980, Fine 1984, Frede 1985 y Crivelli 2004. 
ciado) implica la necesidad (de lo predicado), mientras que el determinismo causal consiste en la tesis de acuerdo con la cual todo evento es producto de causas necesarias (Fine 1984: 23, n. 2). Para el determinismo causal los acontecimientos futuros son necesarios por cuanto se hallan contenidos de antemano en una causa o en una serie de causas, es decir, por cuanto poseen una realidad determinada antes de que efectivamente ocurran. La necesidad de los eventos futuros se funda en un encadenamiento causal fijo e inmodificable que rige todos los acontecimientos. El punto de contacto con el fatalismo estriba en que esta posición presupone que, si una predicción tiene ahora valor de verdad, ello se debe a que el estado de cosas presente es la causa que determina la ocurrencia (o no ocurrencia) del evento futuro y, por lo tanto, constituye indirectamente la causa de su valor veritativo.

Las tradiciones interpretativas antigua y moderna coinciden en denominar "interpretación tradicional" a un conjunto de lecturas que comparten tanto la reconstrucción de la argumentación fatalista como la refutación aristotélica de dicha posición. ${ }^{4}$ En Sobre la interpretación 9, la posición fatalista sostiene el pasaje de la verdad a la necesidad partiendo del Principio de Bivalencia $(\mathrm{PB})$. En otras palabras, si las predicciones son verdaderas, entonces sean necesariamente verdaderas. Según la interpretación tradicional, Aristóteles consideró que se trataba de una argumentación válida pero endeble. Si asumimos que las predicciones se ajustan al $\mathrm{PB}$, entonces la verdad de un enunciado sobre el futuro implica la necesidad de que tenga lugar el evento predicho. ${ }^{5}$ La argumentación fatalista parte de la inalterabilidad del pasado: si una predicción era verdadera en el pasado, entonces siempre fue verdadera y el futuro, en consecuencia, es necesario. ${ }^{6}$ Aristóteles aceptaría la inferencia

\footnotetext{
${ }^{4}$ Entre los especialistas modernos, Sorabji 1980, Frede 1985, van Rijen 1989 y Gaskin 1995 sostienen la interpretación tradicional.

${ }^{5}$ En este sentido, el determinismo sostendría que los valores de verdad de las proposiciones, independientemente de su formulación concreta, son atemporales. En efecto, la enunciación atemporal se condice con la visión determinista (siempre fue verdadera o falsa esta predicción, es decir, el enunciado no cambia su valor de verdad), mientras que la enunciación temporal se corresponde con una concepción no determinista (los enunciados se vuelven verdaderos o falsos de acuerdo con lo que sucede en el curso del tiempo). Para una completa discusión sobre la formulación temporal o atemporal de los enunciados predicativos, véase Sorabji 1980: 96-103.

${ }^{6}$ Según Fine 1984: 23, n. 2, el fatalismo se distingue del determinismo causal en que el último sostiene la tesis de que todo evento está necesariamente causado. Para Fine, Aristóteles rechaza el fatalismo, pero su posición se acerca a un determinismo causal. Aunque rechaza la interpretación tradicional, McKim 1971: 91 considera que el argumento de Sobre la interpretación 18a34-b9 se fundamenta en la necesidad del pasado y del presente. Por su parte,
} 
fatalista porque apela a la necesidad del presente y del pasado: puesto que no podemos modificar el pasado o el presente, no podemos modificar los valores de verdad de los enunciados formulados en un tiempo pretérito o actual.

De acuerdo con la interpretación tradicional, la estrategia aristotélica en Sobre la interpretación 9 para refutar el fatalismo se basa en el rechazo (o la limitación) del PB; en otras palabras, para refutar la necesidad de los eventos futuros, debe negarse que las predicciones sean ya verdaderas o falsas antes de que tenga lugar el evento enunciado. De este modo, las predicciones sobre futuros contingentes carecen de valor de verdad antes de que los eventos que describen tengan lugar, pero pueden volverse verdaderas o falsas cuando el evento predicho ocurre. ${ }^{7}$

Dentro de la interpretación tradicional, podemos distinguir dos variantes en el rechazo al PB: considerar que las predicciones no tienen valor de verdad en absoluto o que esta "neutralidad" sea considerada en sí misma un tercer valor de verdad. En la primera línea, M. Kneale (1962) ha argumentado que Aristóteles rechaza el PB pero mantiene la validez del Principio del Tercero Excluido (PTE). De acuerdo con esta reconstrucción interpretativa, el Estagirita sostendría que las predicciones carecen de valor de verdad, y, en consecuencia, que su valor de verdad no se encuentra fijado de antemano.

En la segunda línea interpretativa, J. Łukasiewicz (1957: 155-156) y A. N. Prior (1953: 317-326 y 1957: 86) sostienen que Aristóteles rechaza tanto el PB como el PTE en el caso de enunciados sobre futuros contingentes: las predicciones no son ni verdaderas ni falsas, sino que poseen un valor de verdad neutral. Para evitar la conclusión fatalista, sostienen que los enunciados sobre futuros contingentes son indeterminados. De acuerdo con Łukasiewicz (1970: 125-126), todas las proposiciones sobre hechos futuros que aún no han ocurrido pertenecen a esta categoría veritativa: las predic-

Hintikka 1973 considera que el fatalismo supone el hecho de que toda verdad actual siempre fue verdadera.

${ }^{7}$ Ackrill 1963: 133, 140-142 mantiene una variante particular de la interpretación tradicional de acuerdo con la cual Aristóteles no rechaza el PB sino que lo cualifica con un "todavía": los enunciados incluidos en una disyunción ("mañana tendrá lugar una batalla naval o no tendrá lugar una batalla naval”) son verdaderos o falsos, pero no todavía (19a36-9), es decir, son veritativamente indeterminados hasta que el futuro ocurra. Según Ackrill 1963: 140, Aristóteles acepta la deducción determinista (si toda predicación es o bien verdadera o bien falsa, entonces todo ocurre por necesidad), pero luego invierte el argumento: puesto que muchos eventos no son aún necesarios (19a7-32), no es necesario que sus respectivas predicciones sean ya verdaderas of falsas (19a32-9). 
ciones no son verdaderas ni falsas en el presente, puesto que carecen de un correlato real que confirme su valor de verdad. No hay nada real en el presente que constituya la causa del evento predicho. Las proposiciones indeterminadas, cuyo correlato ontológico se corresponde con la posibilidad, tienen este tercer valor de verdad. Siendo estas proposiciones indeterminadas, resulta inadmisible atribuirles ningún valor de verdad o de falsedad y, por consiguiente, resulta inaplicable el principio del tercio excluso. En efecto, el Estagirita formula el PTE del siguiente modo: "tampoco puede darse un término intermedio entre los contradictorios, sino que necesariamente se ha de afirmar o negar uno de ellos, sea el que sea, de una misma cosa" (Metafísica IV. 7, 1011b 23-24). En otras palabras, el PTE implica la imposibilidad de que las proposiciones contradictorias que integran una disyunción tengan un valor veritativo diferente de verdadero y falso. Si los enunciados sobre futuros contingentes son indeterminados, entonces escapan a la disyuntiva entre verdad y falsedad.

En línea con la interpretación tradicional, algunos especialistas consideran que la limitación (o rechazo) del PB implica un tercer requerimiento que todo enunciado evaluable veritativamente debe satisfacer: únicamente poseen valor de verdad aquellos enunciados declarativos sobre los cuales puede determinarse si son verdaderos o falsos en relación con el prâgma que tiene por objeto. En esta línea, los enunciados sobre el pasado y el presente satisfacen el requisito de decidibilidad ${ }^{8}$ porque tienen un objeto necesario (y definido), mientras que los enunciados sobre el futuro no cumplen con el requerimiento porque refieren a eventos contingentes (indefinidos). Cabe destacar que los acontecimientos pasados y presentes no se caracterizan por una necesidad simpliciter (algo necesario en cualquier tiempo posible) sino una necesidad presente (aunque su ocurrencia o no es contingente, son necesarios luego de haber tenido lugar). Como veremos en la sección siguiente $(\$ 2)$, la necesidad del acontecimiento presente no es "intrínseca" sino que se deriva de la reciprocidad existencial (Categorías 14b14-2) que mantiene con el enunciado declarativo, es decir, que su necesidad se encuentra esencialmente vinculada con su status de objeto del discurso. ${ }^{9}$

Por otro lado, las interpretaciones no-tradicionales, aunque heterogéneas, se caracterizan por desplazar el eje de la discusión del PB o, incluso,

\footnotetext{
${ }^{8}$ Véase Vigo 2006: 111-112.

${ }^{9}$ El hecho presente no presenta la misma necesidad que el acontecimiento pasado, puesto que no satisface la formulación de "necesario" de Metafísica V. 12, a saber: ser "aquello cuyo contrario es imposible".
} 
admitir la vigencia plena del principio en relación con los eventos futuros. ${ }^{10}$ La pionera en este tipo de lecturas, sin duda fue Anscombe (1956), quien sostuvo que en Sobre la interpretación 9 Aristóteles no suspende la validez del PB sino que introduce la distinción entre verdades necesarias y verdades contingentes. De acuerdo con la intérprete, Aristóteles establece la determinación factual propia del pasado y del presente en contraposición a la contingencia propia del futuro: los valores de verdad asignados a las proposiciones deben reflejar la diferencia ontológica entre estados de cosas pasados-presentes y futuros. En otras palabras, a diferencia del enunciado que tiene por objeto el porvenir, el enunciado relativo al presente es constatable y no indefinido (aóriston).

Por otro lado, Fine (1984) rechaza la interpretación tradicional al sostener que Aristóteles acepta los presupuestos de la argumentación fatalista pero no admite la inferencia porque el pasaje de verdad a necesidad implica una confusión de necessitas consequentiae y necessitas consequentis, ${ }^{11}$ es decir, una falacia de división. La finalidad de distinguir entre dos clases de necesidad estriba en evitar confundirlas: si la necesidad condicional se confunde con la incondicional, se atribuye ilegítimamente necesidad intrínseca al consecuente de un condicional. Para la especialista, esta confusión se encuentra en el centro de la argumentación fatalista: la necesidad de la disyunción "mañana tendrá lugar una batalla naval o no tendrá lugar una batalla naval" es intrínseca, pero deducir la necesidad de una de sus proposiciones es falaz porque les adhiere a estas una necesidad que les es ajena (1984: 29-31). La confusión entre los dos tipos de necesidad transfiere ilícitamente el operador modal del condicional al consecuente. Únicamente los enunciados que tienen por objeto lo que no puede ser de otro modo (porque tiene

\footnotetext{
${ }^{10}$ Entre los antiguos, Boecio y Amonio rechazaron la interpretación tradicional: para estos pensadores, los enunciados sobre eventos futuros son verdaderos o falsos, pero no lo son absolutamente. Para Boecio, la falta de determinación veritativa de los enunciados no se funda en nuestra ignorancia, sino en la naturaleza de esta clase de proposiciones. Véase Boecio, el primer comentario (104 5 - 108 11, 12423 - 126 21) y el segundo a Sobre la Interpretación (13 15, 19928 - 201 2, 2081 - 209 6, 215 21-6, 235 14/17, 245 10-12, 246 12, 24628 - 250 13) y Amonio (CIAG 130 23, 131 3-4, 183 17- 139 20, 141 20, 154 11).

${ }^{11} \mathrm{El}$ primer tipo de necesidad lógica rige los condicionales: si el antecedente es verdadero, entonces el consecuente también debe serlo, pero el consecuente no es, en sí mismo, necesario. Por el contrario, la segunda clase de necesidad se aplica a la proposición consecuente de un condicional. En otras palabras, se trata de una necesidad incondicional (o no cualificada): si $x$ es presente, $x$ es necesario. De acuerdo con Fine 1984: 26, la necesidad cualificada en la silogística es estructuralmente idéntica a la necesidad hipotética en los contextos teleológicos ( $x$ es necesario en función de un fin y al que tiende).
} 
lugar el ámbito supralunar) o lo que ya no puede ser de otro modo (porque ocurrió en el pasado) son necesarios incondicionalmente, mientras que los enunciados que versan sobre los eventos regulares o azarosos son necesarios de forma condicional.

Por su parte, Whitaker (1996:111-112) rechaza la interpretación tradicional al negar que el PB constituya el eje de la discusión en Sobre la interpretación 9, y al afirmar que el fatalismo basa su argumentación en la presuposición de la validez de la Regla de Pares Contradictorios (RPC) respecto de los enunciados futuros: Aristóteles acepta la inferencia fatalista (de la verdad a la necesidad) porque, de acuerdo con la teoría correspondentista de la verdad, todo enunciado verdadero debe tener un referente. Si RPC es válida, entonces la verdad (o la falsedad) de sus proposiciones implica la necesidad de lo predicho por el enunciado, por lo que el futuro es necesario y nada ocurre por azar (18a34-16). Consecuentemente, argumenta Whitaker, la refutación del fatalismo no implica la negación de PB sino de la RPC. ${ }^{12}$

En contraposición a las lecturas fatalistas, podemos hablar de una "interpretación causal" sostenida por algunos especialistas, como Bäck (1992), Von Wright (1984) y White (1981); la argumentación determinista enfatiza la inmutabilidad de los estados de cosas pasados y presentes porque estos ofician de truthmakers (condiciones veritativas) de los enunciados sobre el futuro. Si los estados de cosas pasados y presentes constituyen la causa de los eventos futuros -no pudiendo ocurrir las cosas de otro modo-, entonces estos presentan las condiciones veritativas de las predicciones. De acuerdo con esta lectura, una vez esclarecida la conexión causal entre pasado y futuro, queda en evidencia que la discusión de Sobre la interpretación 9 no versa sobre el fatalismo en sí mismo (el pasaje de la verdad a la necesidad), sino sobre el determinismo causal y su amenaza al libre albedrío. En esta línea, la respuesta aristotélica al determinismo estribaría en negar que los estados de cosas pasados y presentes constituyan truthmakers legítimos de las predicciones.

Por nuestra parte, consideramos que el tratamiento de los futuros contingentes en Sobre la interpretación 9 es, en general, sumamente complejo

\footnotetext{
${ }^{12}$ De acuerdo con Whitaker 1996, una excepción a la RPC no constituye necesariamente una excepción al PB, el cual solo es contradicho por un único enunciado que sea simultáneamente verdadero y falso o que no sea ni verdadero ni falso. Para que la RPC no se cumpla, un par de enunciados contradictorios deben ser, a la vez, verdaderos y falsos: considerados en sí mismos cada uno de los enunciados del par tiene uno y solo un valor de verdad, por lo que cumplen con el PB. En este sentido, de la afirmación de que cada enunciado es o verdadero o falso, no se sigue que esta sea necesariamente verdadera, mientras que de la RPC se deduce que una de las proposiciones que la integran debe ser necesariamente verdadera.
} 
debido a la laxitud con la que el Estagirita expone y conecta sus argumentos, habilitando múltiples y diferentes interpretaciones que, en su pretensión de abarcar la totalidad del capítulo, revelan falencias conceptuales, omisiones textuales o proyectan sobre el pensamiento aristotélico concepciones que le son ajenas. En efecto, entendemos que la dificultosa reconstrucción de los argumentos allí formulados se debe a que Aristóteles no es explícito acerca de la concepción determinista contra la cual discute en el marco dialéctico del capítulo. Nuestra estrategia interpretativa consiste en analizar Sobre la interpretación 9 a partir de nociones y problemas explícitamente abordados por el Estagirita en otras partes del corpus que son relevantes para dilucidar la posición rival y la crítica aristotélica. En líneas generales, acordamos con la interpretación tradicional en que el eje de la discusión es el PB y que su limitación funda el criterio de decidibilidad; sin embargo, consideramos que esta interpretación yerra en considerar que la posición criticada no se identifica con el determinismo sino con el fatalismo. En esta línea, nuestro abordaje de Sobre la interpretación 9 partirá de las nociones de "causa", "naturaleza" y "azar" tal como son conceptualizadas en Física II. 8, 9 y Metafísica. V. 5 y VI. 3 con la finalidad de dilucidar los compromisos fisico-ontológicos de la teoría aristotélica de la verdad predicativa. Dados los límites del presente trabajo, no abordaremos la conceptualización aristotélica del "azar" en Física II. 4 -la cual requiere un análisis en sí mismo- y nos limitaremos al tratamiento de esta noción en conexión con la crítica a la filosofia atomista y al rechazo del determinismo causal. Por otra parte, sostenemos que el Estagirita va cambiando los principios lógicos que emplea en su argumentación, pero siempre manteniendo el $\mathrm{PB}$ como el principio sobre el que se basan los demás.

\section{Planteamiento del problema en Sobre la interpretación 9}

A ristóteles comienza Sobre la interpretación 9 preguntándose si los

A enunciados que tienen por objeto eventos particulares que aún no han ocurrido son verdaderos o falsos (18a28-34). En efecto, el Estagirita formula este interrogante a partir del contraste con los enunciados sobre el pasado y el presente, que necesariamente se ajustan al Principio de Bivalencia (PB): todo enunciado es o bien verdadero, o bien falso. Aristóteles plantea el problema sobre los enunciados futuros de un modo peculiar: a través de la Regla de Pares Contradictorios (RPC): en un par contradictorio de enunciados (antíphasis), necesariamente uno es verdadero y el otro falso. La RPC se funda en la dependencia del valor de verdad de las proposiciones respecto de sus objetos: una afirmación y su negación no pueden ser ambas verdaderas (o ambas falsas) porque no existe un objeto o un evento 
en el plano ontológico que haga simultáneamente verdaderos o falsos ambos enunciados.

En efecto, si toda afirmación o negación es verdadera o falsa, también es necesario que absolutamente todo [lo que se dice], o bien se dé, o bien no se dé. Pues si alguien declara que algo ocurrirá, mientras que otro niega esto mismo, es evidente que uno de los dos estará en lo cierto, si es verdad que toda afirmación es, o bien verdadera, o bien falsa (en efecto, ambas proposiciones no se darán a la vez en tales casos) (Sobre la interpretación 18a34-3). ${ }^{13}$

La verdad predicativa (o por correspondencia) implica una correlatividad: ${ }^{14}$ si el enunciado es verdadero, entonces necesariamente tiene lugar aquello que describe e, inversamente, si tiene lugar lo descrito por el enunciado, este es verdadero; la falsedad se da en el caso contrario (Sobre la interpretación 18a39-b4). Ahora bien, la necesidad del prâgma no consiste en una necesidad intrínseca, sino que se deriva de la reciprocidad existencial (Categorías 14b14-22) que mantiene con el enunciado declarativo: en otras palabras, un objeto o un evento es necesario en tanto correlato del enunciado verdadero, pero no es necesario en sí mismo. El punto es que la correlatividad entre enunciado verdadero y objeto de discurso no implica la necesidad intrínseca del segundo: el evento u objeto es necesario únicamente en tanto correlato del enunciado verdadero. Es por ello que la RPC es introducida en el argumento con fines dialécticos, para robustecer la posición del adversario al garantizar la necesidad intrínseca del prâgma: la verdad y la falsedad se reparten necesariamente entre la afirmación y la negación que componen la contradicción, si lo que ocurre en el plano ontológico es en

\footnotetext{
${ }^{13}$ Seguimos la traducción de Sobre la interpretación de Mittelmann 2009.

${ }^{14}$ Frede 1985: 39-40 señala que el enunciado y el hecho constituyen ítems pertenecientes a la categoría de relativos que (i) presuponen una asimetría: los hechos son anteriores y constituyen la causa de la verdad o la falsedad de los enunciados. Sin embargo, (ii) como el resto de los relativos, son recíprocos en su implicación existencial: si la proposición p es verdadera, entonces es necesario el hecho x y viceversa. Sin embargo, la necesidad en cuestión no es absoluta o causal sino lógica: el hecho es necesario de acuerdo con el presupuesto de que el enunciado que le corresponde es verdadero. De acuerdo con Frede, la necesidad semántica propia de los relativos implica solo que a un valor de verdad definido le corresponde un hecho definido ( $\mathrm{y}$ a la inversa). Esta tesis no suscita dificultades cuando concierne a hechos presentes (que ya están dados), pero cuando se aplica a eventos futuros genera problemas porque estos son contingentes. En suma, para Frede la necesidad lógico-semántica presente en Sobre la interpretación 9 no implica el determinismo sino el fatalismo.
} 
sí mismo necesario. En otras palabras, si la regla refleja una imposibilidad en el plano ontológico -que simultáneamente tengan (o no) lugar los prágmata que corresponden a los enunciados verdadero y falso-, entonces el valor de verdad de sendos enunciados es necesario, porque se deriva de cómo son las cosas. La introducción de la RPC invierte la dirección de la argumentación: no se deduce la necesidad de las cosas en el plano ontológico a partir de la verdad de los enunciados, sino que la necesidad de lo predicho se funda en la necesidad del evento u objeto.

A partir de la necesidad que caracteriza el vínculo entre el enunciado que integra la contradicción y el prâgma, el adversario dialéctico concluye:

De lo que se sigue que nada existe ni acontece -ni existirá o no existirá- ni por casualidad, ni de uno u otro modo al azar, sino que absolutamente todo se da por necesidad y no de una u otra manera (pues o bien el que afirma, o bien el que niega, dice la verdad). Pues de lo contrario todo podría haber ocurrido, ya que lo que puede darse de una u otra manera al azar no es (ni será) más bien de un modo que del otro (Sobre la interpretación 18b5-b9).

En otras palabras, si los enunciados declarativos verdaderos se caracterizan por reflejar un prâgma en el plano ontológico, entonces el objeto futuro, al cual refiere el enunciado verdadero, debe ser necesario en tanto causa antecedente de su valor de verdad. El correlato del enunciado verdadero futuro no puede darse por azar, porque "lo que ocurre de una u otra manera" o "por casualidad" no está fijado o no se encuentra dispuesto de un modo determinado respecto de su ocurrencia. Esto excluye la posibilidad de predicar (afirmativa o negativamente) algo de él; mientras que el prâgma del enunciado verdadero supone precisamente esta posibilidad. Ahora bien, ¿qué clase de necesidad está supuesta en este argumento? ¿Por qué la necesidad es incompatible con el azar?

\section{3. "Necesidad" $y$ "Azar" en Física $y$ Metafísica}

e acuerdo con la definición de "necesidad" de Metafísica, es ne-
cesario aquello que no puede ser de otro modo que como es (1015a33-6). Es por ello por lo que la necesidad excluye la causalidad accidental y el azar, los cuales se caracterizan por la contingencia y la variabilidad. Aristóteles aborda explícitamente el problema de la compatibilidad entre necesidad y azar en su crítica a la filosofía atomista en los capítulos 8-9 del libro segundo de la Física; el Estagirita le adjudica al atomismo tesis aparentemente incompatibles (196a24-35): (i) el azar no es una causa real, (ii) 
todo ocurre por necesidad, y (iii) el azar es la causa del cosmos. ${ }^{15}$ En esta línea, el Estagirita atribuye a Demócrito dos visiones diferentes del azar: por un lado, la ausencia de una causa determinada y, por el otro, la ausencia de una finalidad que guíe los procesos naturales. De acuerdo con la primera visión (195b36-196a5), azar y causalidad son nociones incompatibles, puesto que lo azaroso se vincula con lo inexplicable y, por lo tanto, con la ignorancia de las causas, mientras que la causalidad implica la posibilidad de remontarse a los principios explicativos y a las causas productoras de los fenómenos. En la segunda visión sobre el azar (195b31-33), por el contrario, causalidad y azar son compatibles puesto que este es entendido como una causa, es decir, como la necesidad ciega que resulta de la composición atómico-material de los objetos. En otras palabras, en la interpretación aristotélica del atomismo, el azar es entendido como necesidad porque carece de propósito alguno y es el resultado de una causalidad mecánica. ${ }^{16}$

Ahora bien, la crítica aristotélica al atomismo se centra en el segundo sentido de azar y tiene por finalidad mostrar que lo necesario y lo azaroso se distinguen conceptual y realmente y, a la par, procura distinguir los ámbitos que rigen. En otras palabras, el Estagirita no impugna las nociones de necesidad y de azar, sino que las restringe y las reinterpreta de acuerdo con su concepción teleológica de la naturaleza: ${ }^{17}$ en el ámbito supralunar (el primer motor inmóvil y los astros) rige la necesidad porque el movimiento no se encuentra impedido nunca de alcanzar su finalidad en virtud de la ausencia de materia o de la incorruptibilidad material. En contraposición, en el ámbito sublunar los procesos naturales ocurren con regularidad, pero admiten el azar: la razón de ello es que las entidades sensibles se encuentran compuestas de forma y materia corruptible: la forma se identifica con el fin

\footnotetext{
${ }^{15}$ Véase Rossi 2011: 98-116.

${ }^{16}$ Para complementar la distinción de Física II. 8-9 en torno a la necesidad hipotética y la necesidad “democrítea”, véase De Partibus Animalium I.

${ }^{17} \mathrm{Si}$ bien en algunos contextos específicos el Estagirita emplea el término naturaleza (phýsis) para referirse a la totalidad de los seres y los procesos naturales (Sobre el cielo 290a31, Sobre el alma 432b21), el sentido más relevante y desarrollado en su filosofía natural es el de esencia de una entidad individual. De acuerdo con este sentido, la naturaleza constituye lo más propio de una entidad particular. En Física. II 1 y Metafísica V 4 Aristóteles conceptualiza este sentido técnico exponiendo tres de sus rasgos fundamentales: (1) La naturaleza constituye un principio (arkhê) o causa (aitía), (2) inmanente, (3) del movimiento y reposo de las entidades naturales. Los entes naturales tienen un principio intrínseco de cambio: la noción de "naturaleza", por tanto, se encuentra esencialmente unida a la de "movimiento". En este sentido "naturaleza (...) es la entidad de aquellas cosas que poseen el principio del movimiento en sí mismas por sí mismas” (Metafísica 1015a11-13).
} 
y la materia con el medio para su realización (Física 199a6-31, 199b16-17, 200a25-36). La materia es necesaria para el proceso teleológico únicamente en cuanto medio: su funcionalidad e integración a la entidad natural están determinados por el fin al que tiende, esto es, la forma. ${ }^{18}$ La materia, en cuanto es, a su vez, algo determinado, posee sus propias tendencias naturales que producen algunos procesos específicos en los compuestos sensibles, tales como el decaimiento y la corrupción. ${ }^{19}$

En esta línea, la forma explica la regularidad en los procesos naturales, mientras que la materia daría cuenta de la excepcionalidad de ciertos fenómenos. En efecto, en el octavo capítulo del segundo libro de la Física Aristóteles argumenta contra Empédocles que la presencia de determinadas partes en los animales y el crecimiento de las plantas no tienen por causa el azar sino cierta finalidad:

son por naturaleza todas aquellas cosas que se mueven continuamente en virtud de un principio inmanente y se dirigen a un fin.Y cada uno de estos principios produce un fin diferente para cada cosa (...) Y cuando esto se produce siempre o la mayor parte de las veces no es por accidente ni por azar. En los entes naturales, en cambio, siempre es así si nada lo impide (Física 199b15-25).

Ahora bien, la regularidad en el ámbito sublunar implica también el fenómeno opuesto: los procesos naturales no ocurren ni arriban siempre al mismo resultado - constituyen lo que se da en "la mayor parte de las veces"porque, de hecho, ocurren en la naturaleza fenómenos excepcionales, producto de causas accidentales (Física 196b17-197a1). En los compuestos hilemórficos, la naturaleza material es responsable de los procesos de generación

\footnotetext{
${ }^{18}$ En otras palabras, la forma actúa como causa final en los procesos naturales: su realización implica la necesidad de determinados materiales. Se trata de la llamada "necesidad hipotética", definida en Metafísica 1015a2 como algo sin lo cual el bien no puede realizarse. Desde el punto de vista del fin, la materia es condición necesaria para la realización de la forma. El fin es causa de la materia (es decir, es aquello en vista de lo cual la materia se mueve) mientras que la materia no es causa del fin (vale decir, no es aquello a causa de lo cual el télos se realiza). La forma es causa de la materia en la medida en que esta existe en tanto y en cuanto es un medio y un instrumento para la realización de la primera. Véase Rossi 2011: 74-80.

${ }^{19}$ Estos procesos no se enraízan en la naturaleza formal del compuesto sino en su naturaleza material; dos tendencias contrarrestan las funciones propias de la forma (en tanto principio de identidad y movimiento): por un lado, las partes materiales de los compuestos poseen una inclinación innata a la dispersión y, por otro, la acción de otras entidades materiales involucra al compuesto en procesos que no tienen por causa su forma específica.
} 
y cambio que escapan a la causalidad formal y, por ende, a la regularidad. Ello es lo que queda en evidencia a partir del examen de la generación de monstruos, en donde el resultado del proceso se produce por la primacía de la materia por sobre la naturaleza formal (Generación de los animales 770b15-17). En otras palabras, en los procesos en los cuales la forma no logra dominar la naturaleza material del compuesto, el resultado alcanzado escapa a lo que sucede "la mayor parte de las veces" en los procesos teleológicos orientados hacia la forma como causa final. En suma, en estos casos la materia oficia de causa de aquellos procesos que no obedecen a la finalidad establecida por la forma, y que tienen por fundamento las propiedades materiales propias del compuesto y de los objetos que interactúan con él en el ámbito sublunar. ${ }^{20}$

En MetafísicaVI. 3, Aristóteles expone, aunque con bastante oscuridad, la incompatibilidad de las causas accidentales con el determinismo: estas introducen la contingencia en la cadena de causas necesarias al constituir el punto de partida de aquello que puede ser de un modo u otro (Dudley 2012: 286-314). Allí Aristóteles afirma:

Que hay principios y causas que pueden generarse y destruirse, sin un proceso de generación y destrucción, es evidente. De no ser así, todas las cosas sucederían necesariamente, ya que necesariamente ha de haber una causa no accidental de lo que está en proceso de generación y destrucción (Metafisica 1027a29-32).

En otras palabras, si solo existieran causas per se de todo lo que se genera y se corrompe, entonces todo ocurriría por necesidad. Si este fuese el caso, podríamos retroceder en la cadena causal, eslabón por eslabón, hasta llegar a la causa que produjo lo causado en el presente. Del mismo modo, podríamos deducir de los eventos pasados la necesidad de los eventos futuros. Sin embargo, si retrocedemos al pasado en la cadena causal, nos detendremos forzosamente en algún punto en una causa accidental, cuyos efectos son impredecibles y contingentes. La ocurrencia de estos eventos no presupone la necesidad simpliciter o absoluta que tiene lugar en el ámbito supralunar, sino la necesidad hipotética que la forma imparte sobre la materia en el ámbito sublunar: dado cierto fin (natural o intencional), tales y cuales condiciones materiales han de verificarse si dicho fin ha de alcanzarse; la ocurrencia del

${ }^{20}$ En el fragmento 19a de Sobre la filosofía, trasmitido por Filón de Alejandría, se distingue entre causas internas y causas externas: en algunos casos la accidentalidad se asocia a la materia, pero por factores externos, mientras que en otros la materia es causa natural o interna (no accidental) de los procesos de cambio en virtud de constituir la entidad sensible. 
proceso puede calificarse como regular (si alcanza su fin) o como excepcional (si no lo alcanza).

Por las razones expuestas, no es posible hacer predicciones: puesto que sus causas son contingentes (Metafísica 1027a32-b14), no está determinada la ocurrencia de los eventos en el futuro. ${ }^{21}$ En otras palabras, el futuro no puede predecirse porque la cadena causal no involucra únicamente causas per se sino también causas accidentales, de allí que los eventos futuros sean indeterminados. Recordemos que el carácter accidental de la causa implica que su efecto no se sigue necesariamente de ella, sino contingentemente, y en virtud de otras coincidencias causales.

El azar -tanto en el ámbito de la naturaleza como en el ámbito humano- presupone un trasfondo teleológico: puesto que la regularidad de los procesos tiende a un fin, lo inesperado e irregular consiste en que no se arribe a dicho fin. Todo fenómeno azaroso tiene una causa per se que tiende a un fin regular y esperable. Sin embargo, por sufrir una interferencia causal por parte de causas accidentales, cambia su resultado por otro irregular e inesperado. La interferencia causal se produce en virtud de una coincidencia fortuita y contingente entre la causa per se y otras entidades que, en virtud de la coincidencia, devienen en causas accidentales.

En suma, el carácter excepcional e inesperado del azar lo vuelve impredecible para la ciencia y, a la par,irreductible a la necesidad. ${ }^{22}$ Sin embargo, ello no implica que los fenómenos azarosos sean inexplicables: el evento que ocurre por azar puede explicarse a posteriori apelando a sus causas per se y a sus causas accidentales, una vez que estas han efectivamente operado para producirlo. En esta línea, los eventos pasados y presentes son necesarios y están suficientemente determinados por sus causas en el sentido de que son inmodificables, pero no en el sentido de que su ocurrencia fuese indefectible.

${ }^{21}$ La estrategia aristotélica para refutar la posición del adversario se basa en refutar la necesidad de los eventos futuros, es decir, debe negarse que las predicciones sean ya verdaderas o falsas antes de que tenga lugar el evento enunciado. La contingencia en el valor de verdad de las predicciones refleja la contingencia de la ocurrencia del evento en el futuro. Véase $\$ 1$.

22 En esta línea, Dudley 2012: 316 considera que el determinismo en Aristóteles es epistemológico: "Aristóteles vincula la necesidad (determinismo) con el conocimiento científico. Lo que es determinado/determinable es cognoscible (y, por lo tanto, teoréticamente previsible). Lo que es esencialmente imprevisible no se encuentra predeterminado. Determinado significa "conocido como determinado". Es irracional sostener que cualquier cosa/evento en el futuro es determinado, si no puede ser conocido, no puede ser determinado. En otras palabras, necesidad es una categoría mental". 


\section{La argumentación determinista en Sobre la interpretación 9}

A la luz de lo analizado en el apartado anterior, podemos constatar la Arazón por la cual el Estagirita compara los enunciados verdaderos sobre el pasado y el presente con los enunciados verdaderos sobre el futuro en Sobre la interpretación 9: la causa de la verdad, en el primer caso, es un evento u objeto que es necesario, es decir, inmodificable; mientras que, en el segundo caso, el correlato del enunciado aún no ha ocurrido y es contingente. En otras palabras, la verdad implica necesidad solo en el caso de los enunciados sobre eventos pasados y presentes, pero no en el caso de enunciados sobre eventos futuros. La necesidad de un evento en virtud de sus causas solo se cumple para el caso de lo pasado y lo presente, pero no para el futuro. Aquí "necesidad" y "contingencia" conciernen tanto al plano ontológico como al plano epistémico: puesto que los eventos pasados y presentes ya ocurrieron, no pueden ocurrir de cualquier manera, es decir, excluyen la interferencia del azar y la causalidad accidental (razón por la cual son necesarios); por el contrario, los acontecimientos futuros aún han de ocurrir, admitiendo el concurso de dichas causas (de allí que su ocurrencia no sea necesaria). La necesidad ontológica implica consecuencias inmediatas en el plano epistemológico: en la medida en que las predicciones comportan expectativas epistemológicas, y que tienen por objeto lo contingente, constituyen enunciados impredecibles. En otras palabras, la contingencia ontológica implica que el objeto de las predicciones no pueda conocerse de antemano.

No obstante, analicemos el argumento determinista en Sobre la interpretación 9 , de acuerdo con el cual todos los enunciados, independientemente del tiempo verbal de su formulación, implican necesidad:

Además, si algo es blanco ahora, era verdad decir antes que ese algo será blanco, de modo que siempre ${ }^{23}$ fue verdad afirmar, de cualquiera de los acontecimientos pretéritos que ocurrirá. Pero, si siempre fue verdad decir que un evento es o será, este no podía ocurrir ni dejar de ocurrir en el futuro. Es imposible que no ocurra aquello que no puede no ocurrir y es necesario que ocurra lo que es imposible que no ocurra. En consecuencia, todo lo que ha de ocurrir ocurre necesariamente; de lo que se sigue que nada acontecerá ni de uno u otro modo al azar, ni por casualidad (ya que, si ocurre por azar, no ocurre por necesidad) (Sobre la interpretación 18b9-16)

\footnotetext{
${ }^{23}$ De acuerdo con Hintikka 1973, en este punto la posición determinista desplaza la discusión del plano de la verdad al plano de la necesidad: sea lo que sea que esté sucediendo ahora, era verdad con anterioridad que sucedería.
} 
De acuerdo con esta argumentación, si el pasado es necesario (inmodificable) y las predicciones hechas en ese pasado son verdaderas, entonces el futuro no podía ocurrir de otra manera. ${ }^{24}$ En otras palabras, el enunciado era necesariamente verdadero ya en el pasado, lo que necesariamente determina el acontecer en el futuro. Una argumentación similar puede rastrearse en 18b26-19a1, en la cual se patentiza que el determinista aplica de forma irrestricta el PB y la RPC respecto de los enunciados futuros. En otras palabras: si es necesario que todo enunciado sea verdadero o sea falso (PB), y si es necesario que enunciados contradictorios tengan valores de verdad opuestos (RPC), entonces siempre fue verdadero (o falso) afirmar de los eventos futuros ocurrirán (o no ocurrirán). Sin embargo, la validez de estos principios no implica la necesidad de los eventos futuros. Por el contrario, la posición determinista afirma la simetría entre pasado-presente y futuro, es decir, que la verdad (o falsedad) de los enunciados ya está determinada en virtud de la determinación de los eventos: siempre fue verdad afirmar de los acontecimientos pretéritos que ocurrirían porque estos no podían no ocurrir. De acuerdo con la posición determinista, las predicciones constituyen enunciados como cualesquier otros, susceptibles de ser evaluables veritativamente porque tienen un objeto definido.

Ahora bien, Aristóteles implícitamente sugiere que la argumentación anterior presupone, además de los principios señalados, una premisa más:

Estas consecuencias, y otras similares, son los absurdos que se producen, si (i) es necesario que de toda pareja de afirmación y negación [...] uno de los enunciados opuestos sea verdadero y el otro, falso [RPC]; y (ii) que ninguno de los eventos que actualmente ocurren sucede de un modo cualquiera al azar, sino que todos ellos existan y se produzcan por necesidad. (iii) De manera que no haría falta deliberar ni esforzarse creyendo que, si hacemos tal

${ }^{24}$ Para Jones 2010: 29, la posición fatalista proyecta en sus argumentos la inevitabilidad del pasado y del presente al futuro: los eventos pretéritos y actuales son necesarios porque son inmodificables (es decir, ineluctables). Aristóteles distingue dos clases de necesidad ineluctable: necesidad presente (algo, que pudo haber sido contingente o no, es necesario ahora) y necesidad simpliciter (algo es necesario en cualquier tiempo posible y, por lo tanto, no es que sea necesario en un tiempo en particular). De RPC se sigue que mañana habrá o no habrá una batalla naval, es decir, que, si una de estas proposiciones es verdadera, es necesariamente verdadera. Por lo tanto, si las predicciones eran verdaderas en el pasado, entonces necesariamente debe ocurrir lo que estas predijeron, imposibilitando la multiplicidad de resultados posibles, el azar y la deliberación. De acuerdo con Jones, Aristóteles rechazaría RPC porque conduce a estos absurdos. 
cosa particular, esta ocurrirá, pero, si no lo hacemos, no ocurrirá. ${ }^{25}$ (iv) Nada impide, en efecto, que hace diez mil años alguien haya dicho que esa acción tendrá lugar, y otra persona, que no, de tal suerte que por necesidad ocurrirá cualquiera de las dos cosas que era verdad predecir entonces. (v) Pero no hace ninguna diferencia el que algunas personas efectivamente profirieran los enunciados contradictorios o no, ya que es evidente que, incluso si no fue el caso que uno afirmó y el otro negó, las cosas son como son. Pues no es causa del afirmar o del negar que sucederá o no sucederá un evento, y ello en no mayor medida hace diez mil años que en cualquier otro tiempo (Sobre la interpretación 18b26-19a1).

La argumentación presupone no solo (i) la necesidad lógico-semántica característica de los enunciados declarativos, sino también (ii) la necesidad en el plano ontológico, por lo que la discusión no gira solo en torno al fatalismo sino también al determinismo causal. ${ }^{26}$ En otras palabras, la necesidad de que los miembros del par contradictorio posean valores de verdad opuestos se deriva de una necesidad previa que se encuentra en las cosas mismas. ${ }^{27} \mathrm{~A}$ diferencia de los argumentos anteriores, Aristóteles asume como premisas separadas la validez de la RPC (e, implícitamente, del PB), y la determinación causal de todos los fenómenos. En efecto, si la cadena causal de los acontecimientos es inmodificable, entonces (iv) las predicciones opuestas sobre eventos futuros ya tienen valor de verdad, independientemente de que sea imposible epistemológicamente asignarles los valores que les corresponden. Cabe destacar que al final del pasaje (v) Aristóteles retira

${ }^{25}$ Sobre las consecuencias de la posición determinista-fatalista en el ámbito práctico, el Estagirita afirma: "Sin duda estos razonamientos son inadmisibles. Constatamos, en efecto, que hay un principio de los acontecimientos venideros que provienen tanto de la deliberación como del obrar" (Sobre la interpretación 19a7-9). Dados los límites de nuestra exposición no nos adentraremos en esta crítica. Véase Ética Nicomáquea 1139 b5.

${ }^{26}$ Para White 1981: 240, Sobre la interpretación 9 expone los aspectos semánticos del argumento causal del determinismo de Metafísica VI 3: si lo fortuito no tiene incidencia causal, entonces la ocurrencia o no ocurrencia de todo evento estará predeterminada por lo que ha sucedido y, en consecuencia, todo ocurrirá por necesidad (1027b8-9). Desde una perspectiva semántica, Sobre la interpretación 9 afirma la misma posición: si una proposición sobre el futuro es ahora verdadera (o falsa), ello se debe a que el estado de cosas presente, al cual refiere indirectamente, determina su valor de verdad al determinar la ocurrencia (o no ocurrencia) del evento futuro al que refiere directamente. En esta línea, para Aristóteles el fatalismo y el determinismo causal no serían dos cuestiones separadas sino dos aspectos distintos (lógico y físico) del mismo problema filosófico.

${ }^{27}$ En esta dirección va, precisamente, la conclusión del capítulo en 19 a32-b4. 
momentáneamente de la argumentación la RPC, asumiendo implícitamente que solo con el PB y la presuposición del determinismo causal es suficiente para fundamentar el fatalismo.

Ahora bien, es importante destacar que en el pensamiento aristotélico los principios lógicos son también principios ontológicos o, en todo caso, que obedecen a las determinaciones propias del plano ontológico. En esta línea, en Metafísica Gamma Aristóteles sostiene que el Principio de No Contradicción (PNC) y el Principio del Tercero Excluido (PTE) tienen validez en el plano lógico-discursivo porque tienen por fundamento un plano ontológico que excluye la posibilidad de la contradicción. Del mismo modo, podríamos afirmar que el PB y la RPC se encuentran sujetos a las limitaciones que impone el plano ontológico:

Resulta necesario, por ende, que lo que es sea, cuando es; y que lo que no es no sea, cuando no es. No es necesario, sin embargo, ni que todo lo que es, sea, ni que todo lo que no es, no sea. No es lo mismo decir, en efecto, que "todo lo que es, es necesariamente cuando es", que decir sin restricción: "lo que es, es por necesidad" y la misma distinción debe hacerse, también respecto de lo que no es (Sobre la interpretación 19a23-27).

Entre los eventos que aún no han ocurrido y los que ya sucedieron o suceden tiene lugar una asimetría fundamental: los primeros no se encuentran determinados respecto de su ocurrencia, mientras que los segundos son necesarios, no en el sentido de que eran inevitables antes de su ocurrencia, sino en el sentido de que, una vez que ocurrieron, no pueden modificarse. En consecuencia, los enunciados que integran un par contradictorio tienen asignado un valor de verdad necesario respecto de los eventos presentes y pasados pero, respecto de los hechos futuros, la asignación es variable (pues en este caso, los hechos descritos no han sucedido aún). En otras palabras, el PB y la RPC tienen plena vigencia en lo que respecta a los enunciados sobre el presente y el pasado, es decir, lo inmodificable: su verdad o falsedad es necesaria si los eventos pasados y presentes ya fueron causados. Sin embargo, dichos principios no tienen validez plena en lo que concierne a los enunciados sobre el futuro, puesto que este es indeterminado, es decir, se encuentra abierto ${ }^{28}$ en

${ }^{28}$ De acuerdo con Whitaker 1996: 124-125, los enunciados sobre futuros contingentes poseen valor de verdad, pero se encuentran indeterminados hasta que ocurra (o no ocurra) el evento que predicen. La imposibilidad de saber con antelación cuál miembro del par contradictorio es verdadero o es falso no se funda en un límite del conocimiento humano, sino en la apertura del futuro. 
sus posibilidades de causación, lo que incluye la causalidad accidental y, por ende, la contingencia.

En Sobre la interpretación 19a16-19a23, el Estagirita realiza una distinción interna entre los fenómenos que no son necesarios en relación con su grado de probabilidad: por un lado, se encuentran los eventos azarosos (que pueden o no ocurrir sin ninguna tendencia en absoluto) y, por el otro, fenómenos regulares (que ocurren la mayor parte de las veces). Puesto que los fenómenos regulares ocurren con mayor probabilidad, se vuelven esperables y, por ende, puede decirse impropiamente que uno de los enunciados que integran un par contradictorio es "más verdadero" que el otro. Sin embargo, tal como lo analizamos en la sección anterior de este trabajo, la regularidad no implica necesidad: puede darse una excepción y, en consecuencia, que ocurra el evento al que refiere el enunciado "menos verdadero". En contraposición, en los pares contradictorios que refieren a eventos puramente azarosos, ninguno de los enunciados que lo componen es más o menos verdadero. ${ }^{29}$

Por las razones anteriormente expuestas, la posición fatalista en Sobre la interpretación 9 presupone una concepción determinista: puesto que todos los fenómenos son producto de causas per se, todo ocurre por necesidad, y no puede verse alterada la cadena causal de fenómenos en ningún momento del tiempo. En otras palabras, la argumentación determinista-fatalista puede resumirse en un condicional: si los eventos futuros son necesarios - es decir, son producto de una cadena causal inmodificable--, entonces el PB -y, por ende, la RPC- tienen validez irrestricta.

Aristóteles fundamenta la apertura o la indeterminación del futuro al negar que todas las entidades se encuentren en acto y al afirmar que existe lo potencial:

En general, en las entidades que no están siempre en acto (y en las cuales son posibles ambas cosas: tanto el que existan como el que no existan, e igualmente el que se generen o no), se da lo que puede ser y no ser (Sobre la interpretación 19a9-11).

Esta afirmación debe, no obstante, enmarcarse en la distinción aristotélica entre el ámbito supralunar (donde tiene lugar la necesidad) y el ámbito sublunar (donde tiene lugar la regularidad y el azar). En efecto, puesto que la potencialidad se vincula con la materia y la forma con el acto, lo posible -es decir, aquello que puede o no ocurrir de acuerdo con un propósito-

${ }^{29}$ Véase Física 196b10-17. 
queda excluido del ámbito supralunar. En otras palabras, todo proceso que involucre materia corruptible es susceptible de verse interferido causalmente por causas accidentales y, en consecuencia, de no actualizar la potencialidad de la materia de acuerdo con el fin establecido por la forma. En el ámbito supralunar, las entidades en acto puro excluyen la potencialidad y, en consecuencia, alcanzan necesariamente su fin.

La crítica aristotélica al determinismo, en suma, presupone la apertura del futuro:

Es necesario que todo sea o no sea (y por cierto que necesariamente todo será o no será); pero no cabe dividir diciendo que uno de los dos enunciados de la contradicción sea necesario. Me refiero, por ejemplo, a que necesariamente mañana habrá o no habrá una batalla naval, sin que sea, no obstante, necesario ni que mañana ocurra una batalla naval, ni que no ocurra una batalla naval. Es necesario, en cambio, que o bien ocurra, o bien no ocurra (Sobre la interpretación 19a28-32).

En este argumento Aristóteles no emplea la RPC sino el PTE (la disyunción de una proposición y de su negación es siempre verdadera) y señala que el adversario determinista comete una falacia de división al derivar la necesidad del valor de verdad de los enunciados que componen el par contradictorio (antíphasis) de la necesidad intrínseca del principio en cuestión. ${ }^{30}$ En otras palabras, el PTE es un principio válido y necesario porque refleja, en el plano lógico-discursivo, una necesidad intrínseca al plano ontológico: "mañana ocurrirá una batalla naval o no ocurrirá". ${ }^{31}$ Sin embargo,

${ }^{30}$ Jones 2010: 29-31 se aparta de la interpretación tradicional al sostener que en Sobre la interpretación 9 la refutación al fatalismo implica la cualificación o restricción del PB, la RPC, y el PTE. De acuerdo con el especialista, la argumentación fatalista asume la validez del PTE y aplica la necesidad simpliciter a la disyunción entre pares contradictorios: si es necesario que mañana ocurra una batalla naval o no ocurra, entonces es necesario el valor de verdad de cada una de dichas proposiciones. La falacia de división advertida por Aristóteles se basa, precisamente, en la validez del PTE: de la verdad necesaria de la disyunción, se deduce indebidamente la necesidad de los valores de verdad de los enunciados que integran el par contradictorio.

31 Por nuestra parte, no consideramos que el PTE sea objetado por el Estagirita: que la argumentación falaz del adversario determinista se base en la validez del principio, no es razón suficiente ni legítima para limitar el alcance del PTE. El error, a diferencia del caso del PB y la RPC, no se encuentra en la validez del principio respecto de enunciados futuros contingentes, sino en la inferencia o deducción que parte del PTE y concluye el determinismo. En otras palabras, el principio tiene validez plena respecto de los enunciados 
el operador modal del principio no puede lícitamente distribuirse entre los enunciados que componen la disyunción:"mañana habrá o no habrá una batalla naval" constituye un enunciado necesario, pero la afirmación "mañana habrá una batalla naval" y su negación no comportan valores de verdad necesarios sino contingentes en función de la indeterminación del futuro. Ambos enunciados son potencialmente verdaderos, pero indeterminados en acto y, por esto mismo, contingentes.

Por otro lado, la validez de la RPC respecto de los enunciados futuros es puesta en cuestión por el Estagirita (a los enunciados contradictorios les corresponden necesariamente valores de verdad opuestos), al advertir que de allí no se sigue que la asignación de valores veritativos se encuentre determinada:

En conclusión, dado que las proposiciones son verdaderas según lo sean las cosas, resulta manifiesto que en cuantas cosas sean tales que una u otra de ellas se dé al azar y ambos contrarios sean posibles, es necesario que la contradicción referida a ellas se encuentre en la misma situación.Y esto es precisamente lo que sucede en las cosas que no siempre son o no siempre no son. Pues en estas, es necesario que una de las dos partes de la contradicción sea verdadera o falsa, pero no esta o aquella, sino alguna de las dos al azar y una de ellas resulta verdadera más bien que la otra, pero no es el caso que esta sea ya verdadera o falsa (Sobre la interpretación 19a32-39).

De acuerdo con el Estagirita, el ámbito ontológico impone sobre el enunciado no solo las condiciones de su valor de verdad, sino también las condiciones de su modalidad veritativa: ${ }^{32}$ si el objeto del discurso está sujeto al azar, entonces cualquiera de las alternativas del par contradictorio es posible, con lo cual es imposible determinar de antemano cuál será verdadera y cuál falsa. ${ }^{33} \mathrm{Si}$, por el contrario, el objeto del enunciado no se encuentra

futuros contingentes, porque refleja una necesidad ontológica: es necesario que mañana ocurra o no ocurra una batalla naval, lo cual no implica la necesidad de ninguna de ambas posibilidades ni tampoco presupone que el PB o la RPC se apliquen irrestrictamente.

${ }^{32}$ Esta tesis no es otra que la expuesta en Metafísica, 1051b9-17, donde Aristóteles afirma que la necesidad o la contingencia de las uniones y las divisiones formuladas en el juicio se funda y se corresponde con la necesidad o la contingencia de las uniones y divisiones presentes en el plano ontológico.

${ }_{33}$ Para Whitaker 1996: 124-125, 135 los enunciados sobre futuros contingentes tienen valor de verdad, pero la indeterminación del futuro impide determinar con anticipación cuál es verdadero y cuál es falso. La contingencia de los eventos futuros se refleja en la distribución contingente de valores de verdad entre los enunciados que componen el par contradictorio: como mañana puede ocurrir o puede no ocurrir una batalla naval, entonces la verdad y la 
sujeto a la causalidad accidental, entonces la verdad y la falsedad de los enunciados del par contradictorio es necesaria. Del mismo modo, si el discurso versa sobre fenómenos regulares, los enunciados que componen la antíphasis se diferencian por su mayor o menor grado de probabilidad. En esta línea cierra Sobre la interpretación 9:

Se hace patente así que no es necesario que, de toda afirmación y negación, uno de los opuestos sea verdadero y el otro, falso. Pues en lo tocante a las cosas que no son, pero pueden ser o no ser, la situación no es la misma que de las cosas que son, sino que es tal como se ha descrito (19a39-b4).

Es decir, puesto que el evento que corresponde a los enunciados declarativos futuros es contingente y aún no existe, la RPC no puede aplicarse irrestrictamente, puesto que no puede establecerse con necesidad qué valor de verdad le corresponde a cada miembro del par contradictorio. Su validez se restringe a establecer, en abstracto, que enunciados contradictorios poseen valores de verdad opuestos sin poder determinar el valor de verdad que le corresponde a cada uno. En contraposición, la regla se aplica sin restricciones a los prágmata que son necesarios (como el movimiento de los astros) o aquellos que, siendo contingentes, ya han ocurrido y, por ende, no pueden modificarse: allí puede establecerse fácilmente el valor de verdad correspondiente a cada miembro de la antíphasis.

Ahora bien, la limitación de la RPC se funda en la limitación previa que cualquier enunciado tiene (independientemente de su integración en un par contradictorio), es decir, la restricción del PB respecto de los eventos futuros se debe a que la determinación veritativa no puede efectuarse en virtud de la indeterminación ontológica del objeto de discurso. En otras palabras, si la verdad o la falsedad se establecen en virtud de la relación entre enunciados (o juicios) y prágmata, entonces la ausencia del objeto imposibilita la relación y, por ende, la posibilidad de asignar valores veritativos a nuestro discurso (o juicio). ${ }^{34}$

falsedad de sus respectivos enunciados no se encuentra determinada. Si no se puede asignar verdad y falsedad a los enunciados en cuestión, entonces RPC no se aplica con validez a las predicciones. Consideramos que la lectura de Whitaker sobre la apertura del futuro es acertada, pero yerra en considerar que la solución aristotélica no implica el rechazo de PB, puesto que RPC presupone dicho principio. De acuerdo con Jones 2010, la posición de Whitaker es inconsistente: ¿cómo pueden los enunciados sobre el futuro tener valor de verdad y ser, a la vez, indeterminados?

${ }^{34}$ De acuerdo con Byrd 2010, quien sostiene una perspectiva semántica de la interpretación 


\section{Conclusiones}

A lo largo de nuestro análisis de los pasajes centrales de Sobre la in-

Aterpretación 9, procuramos explicitar los presupuestos físico-ontológicos de la posición contra la cual discute Aristóteles, y concluimos que el adversario sostiene una concepción determinista, la cual involucra distintos principios lógicos en diferentes fases de la argumentación: (i) En el comienzo del capítulo (18a34-39, 18b5-b9), el pasaje de la verdad a la necesidad se sostiene por medio del PB y la RPC, es decir, el fatalismo se argumenta desde la necesidad lógico-semántica propia de los enunciados declarativos (PB) y la necesidad en el plano ontológico (RPC). Sin embargo, la argumentación comporta consecuencias inmediatas en el plano ontológico, puesto que nada sucede en virtud de causas accidentales sino de causas per se, no hay lugar para el azar porque todo ocurre por necesidad (incluso los hechos futuros). En la siguiente fase (ii), el adversario prescinde de la RPC y sostiene el pasaje de la verdad a la necesidad invirtiendo la argumentación (18b26-19a1): si el futuro ya se encuentra determinado, entonces los enunciados sobre el futuro no solo poseen valores de verdad, sino que estos son necesarios. La vigencia plena del PB (y también de la RPC) sobre eventos futuros se fundamentaría en que estos, al igual que los hechos pasados y presentes, son inmodificables. Finalmente (19a28-32), el adversario determinista argumenta el pasaje de la verdad a la necesidad por medio del PTE: de la necesidad intrínseca de una disyunción que involucra enunciados opuestos sobre el futuro, infiere la necesidad de cada miembro del par.

Correlativamente, la crítica aristotélica a la posición determinista varía de acuerdo con los principios lógicos involucrados en la argumentación del adversario: el Estagirita explícitamente rechaza sus supuestos, es decir, niega que el PB y la RPC tengan plena vigencia respecto de los enunciados futuros puesto que, de ser válidos, se deduce la imposibilidad del azar y del libre albedrío. Ahora bien, Aristóteles niega que todo ocurra por necesidad, puesto que en la causación de cualquier evento (pasado, presente o futuro) interviene la causalidad accidental (Física 196b17-197a1).

tradicional, el PB es una consecuencia directa de la teoría adecuacionista de la verdad y es rechazado por Aristóteles porque los enunciados sobre futuros contingentes no son aún verdaderos o falsos, es decir, dependen de un truthmaker que aún no existe, lo cual equivale a decir que el enunciado carece de condiciones veritativas. El Estagirita acepta la inferencia fatalista porque, si existiese el evento futuro, este determinaría el valor de verdad del enunciado, es decir, su valor de verdad sería necesario. 
Los eventos futuros se diferencian de los pretéritos o actuales en que se encuentran abiertos en sus posibilidades de causación, es decir, son pasibles de ocurrir debido a causas accidentales, de allí que el PB y la RPC restrinjan su validez respecto a enunciados sobre el futuro. En otras palabras, de acuerdo con la argumentación determinista, no hay asimetría entre los enunciados que versan sobre el pasado-presente y el futuro: ambas clases de enunciados son necesarios en el sentido de que se encuentran suficientemente determinados por sus causas. La necesidad de su objeto no estriba en que arriba indefectiblemente a su causa final, sino en que son eventos inmodificables. La crítica aristotélica pretende mostrar la asimetría: los eventos futuros no son indefectibles, sino contingentes, porque admiten la intervención de causas accidentales que modifican el proceso hacia un resultado diferente e imprevisto.

En esta línea, destacamos que la necesidad queda limitada al ámbito supralunar y que la regularidad y el azar tienen lugar en el ámbito sublunar: en efecto, la materialidad que caracteriza a las entidades sensibles sublunares involucra la causa accidental en virtud de la cual los procesos naturales no siempre arriban al mismo resultado (Generación de los animales, 770b15-17), es decir, debe buscarse en la materia y no en la forma la causa en virtud de la cual la actualización de la materia no arriba al fin esperado y determinado formalmente (Física 199a6-31, 199b16-17, 200a25-36).

Por otra parte, Aristóteles no objeta la validez del PTE en la argumentación determinista pero sí rechaza la inferencia que deduce la necesidad de los enunciados que integran una disyunción de la necesidad intrínseca de la disyunción en cuestión.

En suma, puesto que los enunciados sobre futuros contingentes carecen de un correlato objetivo en virtud del cual se determina su valor de verdad, el PB no puede aplicarse de forma irrestricta a enunciados que refieren a prágmata indeterminados. Esta restricción funda el criterio de decidibilidad: no son evaluables veritativamente aquellos enunciados cuyo valor de verdad no puede determinarse en relación con su objeto de discurso. En otras palabras, un enunciado cumple el criterio de decidibilidad si puede aplicarse el PB al vínculo que mantiene con su objeto en el plano ontológico. En esta línea, la limitación del PB (y la RPC) con respecto a los enunciados sobre hechos futuros no se funda únicamente en la necesidad (o contingencia) lógico-semántica que caracteriza al discurso y al juicio en su relación con la realidad, sino también -fundamentalmente- en la necesidad (o contingencia) propia del ámbito ontológico que constituye el correlato de los enlaces lógico-lingüísticos. 


\section{BIBLIOGRAFÍA}

Ackrill, J. (1963), Aristotle, Categories and De Interpretatione, traducción y comentario (Oxford: Clarendon Press).

Alejandro de Afrodisia, De Fato, trad. R. W. Sharples (London: Duckworth, 2003).

Anscombe, G. E. M. (1956), "Aristotle and the Sea Battle”, Mind, 65: 1-15.

Aristóteles, Categorías, Sobre la interpretación, traducción, introducción y notas de J. Mittelmann (Buenos Aires: Losada, 2009).

Aristóteles, Física (Libros I y II), traducción, introducción y notas de M. Boeri (Buenos Aires: Biblos, 1994).

Aristóteles, Metafísica, traducción y notas de T. Calvo Martínez (Madrid: Gredos, 1994).

Bäck, A. (1992), "Sailing through the Sea Battle", Ancient Philosophy, 12: 133-151.

Barnes, J. (1984) The Complete Works of Aristotle, Princeton: University Press, 2 vols.

Meiser, C. (1880), Commentarii in Librum Aristotelis ПEPI EPMHNEIA $\Sigma$ (Leipzig:Teubner).

Byrd, J. (2010), “The Necessity of Tomorrow's Sea Battle”, The Southern Journal of Philosophy, 48(2): 160-176.

Cicerón, De Natura Deorum: Liber I, trad.A. R. Dyck (Cambridge: Cambridge University Press, 2003).

Cicerón, On the Orator: Book 3. De Fato. Stoic Paradoxes. Division of Oratory, trad. H. Rackham (Cambridge: Loeb Classical Library, 1942).

Crivelli, P. (2004), Aristotle on Truth (Cambridge: Cambridge University Press).

Diógenes Laercio, Lives of Eminent Philosophers, trad. P Mensch y ed. J. Miller (Oxford: Oxford University Press, 2018).

Dudley, J. (2012), Aristotle's Concept of Chance: Accidents, Cause, Necessity and Determinism (New York: State University of New York).

Fine, G. (1984), “Truth and Necessity in De Interpretatione 9”, History of Philosophy Quarterly, 1: 23-47.

Frede, M. (1985), “The Sea-Battle Reconsidered:A Defense of the Traditional Interpretation", Oxford Studies in Ancient Philosophy, 3: 31-87.

Gaskin, R. (1995), The Sea Battle and the Master Argument (New York:Walter de Gruyter).

Hintikka, J. (1973), Time and Necessity: Studies in Aristotle's Theory of Modality (Oxford: Oxford University Press).

Jones, R. E. (2010), “Truth and Contradiction in Aristotle's De Interpretatione 6-9”, Phronesis, 55(1): 26-67.

Kalbfleisch, C. (1962) (ed.), Simplicii in Aristotelis Categorias commentarium, Commentaria in Aristotelem Graeca, vol. 8 (Berlin: De Gruyter).

Kneale, M. (1962), The Development of Logic (New York: Clarendon Press).

Lukasiewicz, J. (1957), Aristotle's Syllogistic: From the Standpoint of Modern Formal Logic (Oxford: Oxford University Press).

Łukasiewicz, J. (1970), "On Three-Valued Logic", en L. Borkowski (ed.), Jan Łukasiewicz: Selected Works (Amsterdam: North-Holland). 
McKim, V. R. (1972), "Fatalism and the Future: Aristotle's Way Out", Review of Metaphysics, 25: 80-111.

Prior, A. N. (1953), "Three-valued Logic and Future Contingents", Philosophical Quartely, 3 (13): 317-326.

Prior, A. N. (1957), Time and Modality (Oxford: Oxford University Press).

Pseudo Plutarco, Plutarch's Moralia, Volume VII (On Love of Wealth. On Compliancy. On Envy and Hate. On Praising Oneself Inoffensively. On the Delays of the Divine Vengeance. On Fate), trad. P. H. De Lacy \& B. Einarson (Cambridge-Massachusetts: Harvard University Press, 1959).

Ross, W. D. (1979) Aristotle, Physics. A Revised Text with Introduction and Commentary (Oxford: Clarendon Press).

Rossi, G. (2011), El Azar Según Aristóteles: Estructuras de la causalidad accidental en los procesos naturales y prácticos (Sankt Agustin:Verlag).

Sorabji, R. (1980), Necessity, Cause, and Blame: Perspectives on Aristotle's Theory (Ithaca: Cornell University Press).

Van Rijen, J. (1989), Aspects of Aristotle's Logic of Modalities (Dordrecht-Boston-London: Kluwer Academic).

Vigo, A. (2006), Estudios aristotélicos (Pamplona: Universidad de Navarra).

Von Wright, G. H. (1984), Philosophical Papers of Georg Henrik Von Wright,Vol. 3 (Oxford: Basil Blackwell).

Whitaker, C. W. A. (1996), Aristotle's De Interpretatione: Contradiction and Dialectic (Oxford: Clarendon Press).

White, M. J. (1981) "Fatalism and Causal Determinism”, Philosophical Quarterly, 31:231241.

Recibido: 13-05-2020; aceptado: 25-08-2020 